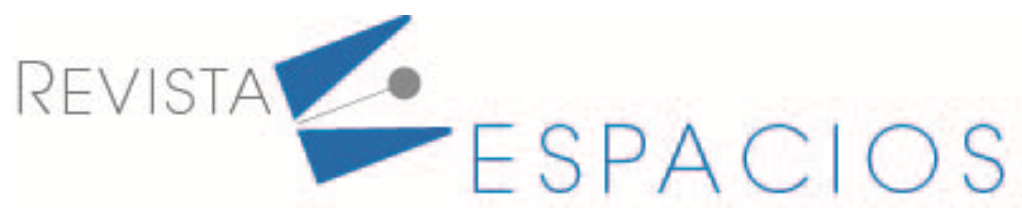

\title{
Gestión de la responsabilidad social: equilibrio para la sostenibilidad en la administración pública
}

\section{Social responsibility management: balance for sustainability in public administration}

\author{
MOROCHO, Zaida P. ${ }^{1}$
}

\begin{abstract}
Resumen
El presente documento realiza una aproximación desde la perspectiva de la gestión de responsabilidad social en la administración pública y su grado de corresponsabilidad en las prácticas de buen gobierno. Para ello, se realiza un estudio de tipo documental, con enfoque cualitativo a partir del análisis bibliográfico de normas y definiciones establecidas sobre el tema. Con los resultados de la investigación se propone un esquema de gestión responsable y desarrollo sostenible para el equilibrio en la administración pública del Ecuador.
\end{abstract}

Palabras clave: responsabilidad social, gestión pública, sostenibilidad

\begin{abstract}
This document makes an approach from the perspective of social responsibility management in public administration and its degree of co-responsibility in good governance practices. For this, a documentarytype study is carried out, with a qualitative approach based on the bibliographic analysis of norms and definitions established on the subject. With the results of the research, a responsible management and sustainable development scheme is proposed for balance in the public administration of Ecuador.

Key words: social responsibility, public management, sustainability
\end{abstract}

\section{Introducción}

El artículo analiza la gestión de la responsabilidad social como estrategia para el equilibrio de la sostenibilidad en la administración pública, ante los desafíos de la globalización y modernización. A partir de la conceptualización de desarrollo sostenible y responsabilidad social, el estudio plantea el análisis de las aplicaciones y beneficios que ofrece la administración pública como respuesta a las necesidades y exigencias de la sociedad civil ante las instituciones del Estado. Lo que conlleva a este último a asumir un comportamiento socialmente responsable tanto en el ámbito interno como externo, para alcanzar un equilibrio entre la gestión responsable y la sostenibilidad de la administración frente a los nuevos desafíos que deben enfrentar a nivel mundial.

El concepto de responsabilidad social ha sido limitado a las acciones que emprenden las entidades, especialmente a aquellas que tienen efectos e impacto en el entorno de las mismas. Sin embargo, la responsabilidad social tiene un campo de aplicación mucho más amplio, no solo en el entorno de las entidades sino también al interior de las mismas, en cada actividad, actitud y comportamiento de los individuos en el ejercicio de sus funciones habituales como valor en la cultura organizacional (Gaete, 2008).

${ }^{1}$ Docente. Universidad Técnica de Machala.zmorocho@utmachala.edu.ec 
La visión de la responsabilidad social en el marco público, plantea la necesidad de establecer una gestión basada en principios y valores que permitan desarrollar una relación con la sostenibilidad para armonizar la administración pública. Esta relación expresará una preocupación constante en los impactos generados por las actividades desarrolladas en cada uno de los sistemas de gestión de una institución.

En la actualidad, el estado ha desarrollado importantes procesos de modernización de la gestión pública, incorporando técnicas desarrolladas desde el sector privado, en este escenario, surge para la gestión pública un desafío adicional a partir de la aproximación conceptual del desarrollo sostenible y responsabilidad social, con la necesidad de diferenciarse y establecer ventajas competitivas en organizaciones similares desde la gestión responsable, fortalecer la imagen corporativa ante la sociedad y la confianza en las administraciones públicas, alineadas con la sociedad donde se incluyen los stakeholders fomentando la responsabilidad ética en la sociedad (Cueto \& De la Cuesta, 2016).

Con base en lo descrito, se puede afirmar que la responsabilidad social en la administración pública, debe concretarse a nivel de políticas públicas integradas por procedimientos de gestión, de contratación, de consumo, recursos humanos y monetarios; transformándose esta gestión en indicadores que permitan evaluar la contribución y la excelencia de la administración pública tendiente a la mejora continua frente a la sociedad.

En este sentido, el objetivo de la investigación es analizar la gestión de responsabilidad social como estrategia para el equilibrio de la sostenibilidad en la administración pública y su relación directa con los objetivos de desarrollo sostenible; utilizando como metodología la revisión documental y bibliográfica de los aportes existentes, el análisis de la instrumentalización de la responsabilidad social dentro de la gestión pública a través de normas, estándares globales y modelos de excelencia; identificando la gestión responsable como un elemento clave para que la administración pública pueda enfrentar de manera eficiente los nuevos desafíos para el desarrollo sostenible.

\subsection{Desarrollo teórico}

El papel del sector público no debe limitarse al cumplimiento del marco normativo y en su caso de la exigencia de responsabilidades por sus acciones y aplicación de modelos de gestión. El "deber ser" abarca el ámbito de las expectativas y los valores sociales, que sin estar normados se encuentran intrínsecamente ligados a expresar aceptación o rechazo de determinadas conductas y comportamientos de las entidades públicas y sus administradores.

Resulta adecuado iniciar con el análisis del concepto de gestión pública, citando a Albi, González y López, quienes afirman que la gestión pública es "un conjunto de decisiones de coordinación y motivación de las personas plasmados en procedimientos y mecanismos contractuales, para alcanzar los fines de la organización estatal (eficiencia y equidad), dentro de las restricciones del marco jurídico-político". Los autores resaltan en su análisis el concepto de gestión, entendiendo por tal "un conjunto de reglas y decisiones dirigidas a incentivar y a coordinar acciones", y el concepto de pública debido a que "persigue metas colectivas y se desenvuelve en el marco de unas restricciones jurídico-políticas peculiares” (Albi, González, \& López, 1997, págs. 20-21).

Desde la perspectiva de Gaete (2008), el concepto de gestión pública también se vincula con el enfoque de las políticas públicas hacia temas relacionados con la salud, educación o vivienda, las que representan necesidades sociales, demandando gestión pública eficaz para lograr satisfacerlas, sobre todo en lo relacionado a las decisiones que permitan desarrollar políticas públicas pertinentes.

De acuerdo con lo anterior, una gestión pública responsable, es efectiva cuando se basa en la correcta adopción de políticas y eficiente administración de los recursos del Estado, con la finalidad de satisfacer las necesidades de la ciudadanía e impulsar el desarrollo del país. 
Tomassini (1994) señala que para una acertada elección y ejecución de políticas públicas "los países deberán incorporar muchos de los aportes provenientes de la moderna teoría de las organizaciones, de los conocimientos logrados en materia de gestión o management sciences". Bajo este contexto, reafirma la importancia que tiene el hecho de que los servicios públicos adopten una nueva gestión pública para el adecuado desarrollo de sus políticas, enfocándose hacia la obtención de los mejores resultados, mediante la utilización de técnicas modernas de gestión y participación de la ciudadanía en los procesos de gestión.

Para lograr un Estado eficiente ante los desafíos y requerimientos de la sociedad civil, se hace necesario, además del nuevo concepto de gestión pública, la restructuración del Estado para que pueda responder con oportunidad, pertinencia, calidad y eficacia frente a la sociedad civil a través de sus políticas, haciendo necesaria la participación activa de los ciudadanos en la selección, formulación y ejecución de dichas políticas públicas para beneficio común (Tomassini \& Armijo, 2002).

Un aspecto importante de analizar junto con los desafíos y requerimientos para lograr un estado eficiente, son los obstáculos que la gestión pública debe sortear especialmente en Latinoamérica, es la resistencia al cambio ante los procesos modernizadores que en su mayoría afectan a los procesos de gestión pública, sobre todo en lo relativo a la participación ciudadana en el accionar del Estado, tanto de sus servicios públicos como en sus políticas y programas públicos. (Gaete, 2008, p.44).

Hoy en día, es admisible la oportunidad de gestionar los recursos públicos, no solo lo enmarcado legalmente, sino también con la oportunidad de innovar esta gestión mediante otras técnicas e instrumentos, acorde a las buenas prácticas de gestión responsable en las administraciones públicas, especialmente las relacionadas de forma directa con los ciudadanos (Cueto, 2009).

Existe todo un marco normativo, códigos éticos y de buen gobierno para fomentar la práctica de políticas responsables de forma obligatoria en el contexto de las administraciones públicas; así como índices asumidos de forma voluntaria para evaluar la transparencia y la excelencia socialmente responsable y sostenible.

Las políticas públicas se encuentran relacionadas de forma directa con la responsabilidad social ya que es la manera que tiene el Estado para dar respuestas o soluciones a las demandas de la sociedad, con la finalidad de promover mejores estándares sociales, económicos y medio ambientales. Fox, Ward, \& Howard (2002) afirman:

Las políticas públicas en materia de responsabilidad social corporativa se clasifican en:

- Políticas reguladoras del sector público, mediante la cual se definen los estándares mínimos de la actuación empresarial dentro del marco legal.

- Políticas facilitadoras o de apoyo, que permiten estimular la implicación de actores clave a través de la puesta en marcha de formación, comunicación o de difusión.

- Políticas de colaboración y acuerdos estratégicos, pueden agrupar y poner en común las capacidades y los logros de los sectores públicos y privado y la sociedad civil.

- Políticas de promoción o reconocimiento directo de los esfuerzos de otras organizaciones, o el mismo efecto ejemplarizante que tienen las prácticas de gestión del sector público. (p.6)

El impacto de estas políticas no sólo se entiende en la relación tradicional de la acción de los gobiernos hacia el sector privado, sino que permite la existencia de una relación multilateral y puede orientarse a su integración en la propia administración pública, en la relación entre gobierno y sociedad civil; o en una relación multilateral entre gobiernos, empresa y sociedad civil (Lozano, Albareda, Tamyco, Roscher, \& Marcuccio, 2005).

Generalmente, el análisis que se hace sobre responsabilidad social se ha centrado básicamente en la adopción de modelos de gestión empresarial haciendo énfasis en las acciones ejecutadas en los ámbitos económico, social 
y ambiental; con responsabilidad en el impacto que se deriva de dichos actos hacia los grupos de interés, medibles mediante la rendición de cuentas, la observación y seguimiento de los modelos externos de gestión empresarial. No obstante, un campo importante de reflexión y debate es su relación y articulación con las políticas públicas. Tal como lo señala Lafuente, et al. (2003), la discusión sobre la responsabilidad social se ha trasladado del ámbito empresarial al de las políticas públicas, principalmente en lo relacionado con la promoción de la filosofía de la responsabilidad social desde los poderes públicos con el propósito de favorecer las ventajas competitivas y de reputación que esta implica para las empresas que la incorporan.

La condición general es que las organizaciones ejercen su responsabilidad social cuando su visión enmarca las expectativas que tienen sus diferentes grupos de interés, acerca de sus comportamientos. En el contexto de una nueva gestión pública, la intersectorialidad entendida como el trabajo coordinado con otro tipo de organizaciones (empresas, organizaciones sociales y ciudadanas) y otras instituciones estatales, promueve la conformación de redes que optimizan el uso de recursos y enfoques, dirigiéndoles en forma eficiente hacia la satisfacción de las necesidades de la sociedad. Al mismo tiempo, los cambios en las demandas y aspiraciones de la sociedad civil, hacen necesario incorporar la "transversalidad" en la implementación de la política pública, a partir de la generación de buenas prácticas, en un modelo de responsabilidad social que se caracterice por atender estas nuevas demandas como variables dinámicas en los procesos de gestión pública (Zenck, Ríos, Pogo, \& Cueto, 2017).

Actualmente el modelo de gestión para la responsabilidad social se ha trasformado, desde un modelo donde los ciudadanos son clientes y consumidores, a otro donde son socios, capaces de participar (Navarro, Ruíz, De Los Ríos, \& Tirado, 2014). Analizando esta teoría, los autores indican que la responsabilidad social de las organizaciones no debería estar basada en logros, sino en relación con las demandas de los stakeholders, esto con el propósito de dirigir esfuerzos de una manera más organizada, eficiente y oportuna, convirtiéndolos en participantes de un cambio. También se presenta una gran modificación al interior de la organización, teniéndose que implementar políticas de responsabilidad social en los procesos de planificación, considerando el punto de vista de los grupos de interés involucrados.

La implantación de la responsabilidad social debe ser un proceso de mejora y concienciación continúa basándose en la importancia de la sostenibilidad, revisando los principios y conceptos sobre responsabilidad social hasta movilizar a la organización hacia una nueva cultura, donde se destaca la participación de los grupos de interés. El propósito de la gestión de la responsabilidad social es alinear la organización con las demandas dinámicas de los negocios y de los stakeholders sobre aspectos sociales y medioambientales a través de 4 etapas clave (Maon, Lindgreen \& Swaen, 2009):

Cuadro 1

Etapas clave en la gestión de la Responsabilidad Social

\begin{tabular}{l}
\hline Redefinir los conceptos sobre responsabilidad social (planificación) \\
\hline Movilizar la gestión hacia la responsabilidad social (ejecución y evaluación) \\
\hline Senstaurar una nueva cultura corporativa (implantación) \\
\hline Fuente: (Maon, los miembros de la organización sobre la responsabilidad social (concienciación)
\end{tabular}

Fuente: (Maon, Lindgreen, \& Swaen, 2009)

La gestión responsable para Cueto \& De La Cuesta (2019) desde la planificación, debe cumplir la normativa legal y atender al contexto en el que desarrolla su actividad, de lo contrario carece de legitimidad y sostenibilidad en el tiempo. Siendo el entorno público un escenario donde se interrelacionan aspectos como la protección medioambiental, los Derechos Humanos, la protección a la salud y a los consumidores, la ayuda a la comunidad, el respeto a las reglas del mercado y el desarrollo social, las políticas fiscales, la transparencia y rendición de 
cuentas e impactos económicos, sociales y ambientales; afectando estos de manera directa a los distintos stakeholders ante los que se responde (empleados, clientes, proveedores, inversores, etc.).

En principio, la aplicación de las políticas públicas deben aplicarse desde el interior de las entidades; si se cumple con los requerimientos legales, se aplican valores éticos, y se trabaja con transparencia, se transmite mayor confianza a los proveedores, clientes, la sociedad en su conjunto; además de la evaluación de sus resultados para garantizar la mejora continua, de acuerdo como se visualiza en la figura 1, para Cueto \& De la Cuesta (2016), que permite integrar la responsabilidad social en la gestión empresarial, y por otro dotar a esta de carácter estratégico que se despliegue por fases.

Figura 1

Proceso de mejora continua en la gestión de la RSC

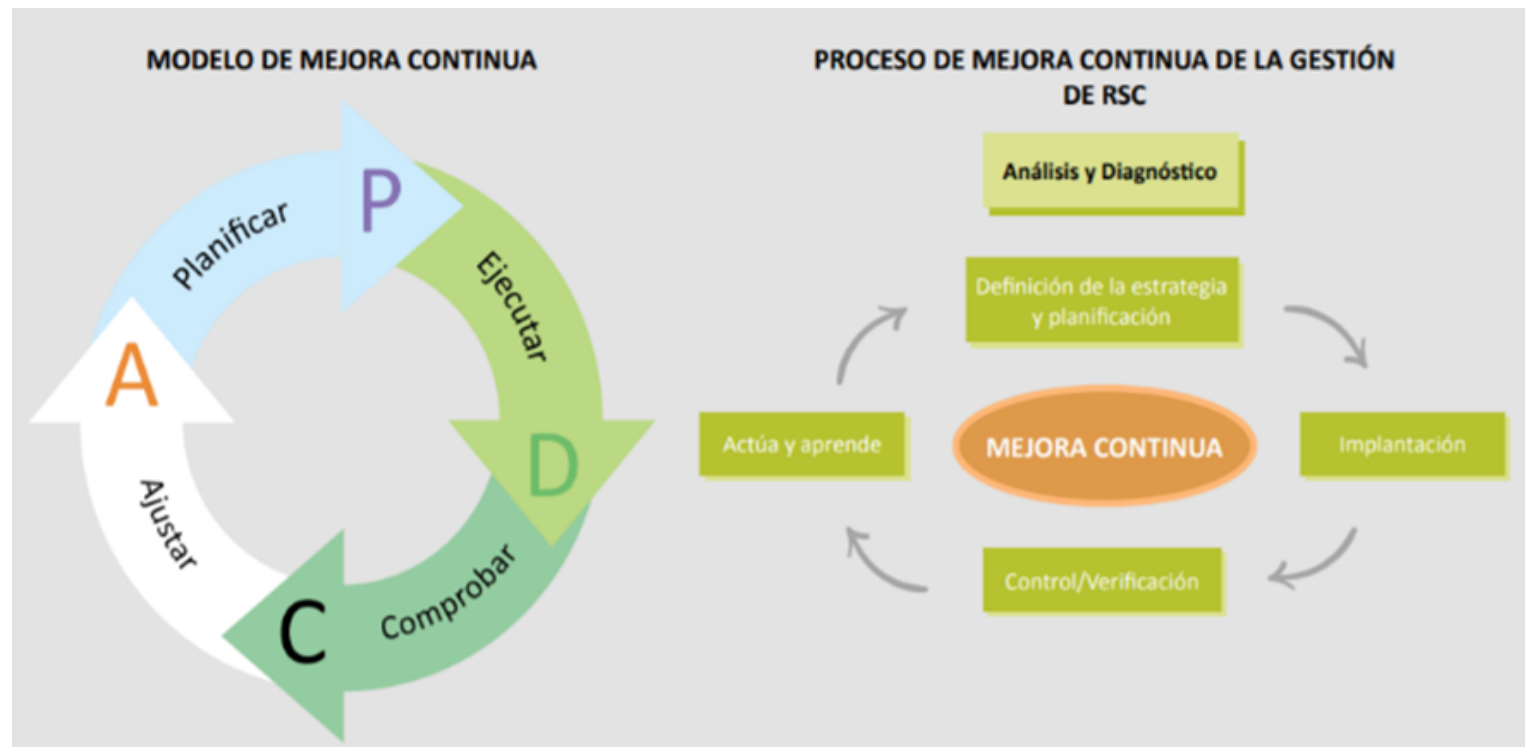

Fuente: Cuesto \& De La Cuesta (2016)

El proceso de Mejora Continua es un elemento de evaluación en las organizaciones tanto públicas como privadas. Prácticamente, desde los años noventa el concepto de procesos ha irrumpido las bases del management (Biazzo \& Benardi, 2003). Garvin (1998) indica, al respecto, que dicho entusiasmo por los procesos por parte de las organizaciones fue el resultado de tener la posibilidad de reducir la fragmentación del trabajo por departamentos y mejorar la capacidad de coordinación y comunicación transversal.

Al respecto, Suarez-Barraza \& Ramis-Pujol (2008) afirman: "la Mejora Continua de Procesos es aplicada en la gestión de las administraciones locales mediante actividades de mejora, las cuales se integran en un conjunto de etapas evolutivas en diferentes niveles de aplicación" (p.80).

De acuerdo con lo expresado, el mejoramiento continuo es un principio básico de la gestión pública, donde la mejora continua debería ser un objetivo estratégico permanente a fin de incrementar gestión responsable de los servicios públicos, programas estratégicos, proyectos y obras públicas; beneficiando a los grupos de interés y ciudadanía en general.

Para Gaete (2008) otro de los elementos que fortalece un comportamiento socialmente responsable, es la rendición de cuentas (accountability) y la transparencia expresada a través del pleno acceso a la información, por ejemplo, en la publicación de memorias de sustentabilidad o ejecución presupuestaria. Resulta evidente que por sí mismas la transparencia y la rendición de cuentas se transforman en aspectos característicos y distintivos de la gestión pública, sin embargo, también se transforman en indicadores de un comportamiento socialmente responsable, razón por la cual es importante no considerar como sinónimos a la transparencia y la rendición de 
cuentas con la responsabilidad social, ya que las primeras son expresión de un aspecto específico del proceso de gestión y toma de decisiones de una organización (la evaluación y el control por ejemplo), en cambio la responsabilidad social debe abarcar y trascender a todas las fases y actividades de la gestión de una organización.

La revisión literaria relacionada con las políticas públicas que promueven prácticas de responsabilidad social, permite identificar que estas se han generado en escenarios internacionales. Haciendo un recorrido de inicio por la Unión Europea con la presentación del Libro Verde en el año 2001, con lo que se constituye un referente para la definición de responsabilidad social, de esta iniciativa se articulan los lineamientos de la Organización para la Cooperación y Desarrollo Económico (OCDE), el Pacto Global de las Naciones Unidas y el modelo del Global Reporting Initiative (GRI).

Las contribuciones de la Comisión Europea (CE) a través del Libro Verde se enmarcan en la naturaleza multistakeholder, (representantes de empresarios, trabajadores, consumidores, asociaciones profesionales, redes de empresas y la sociedad civil); el mismo que se ha convertido en un referente para el debate y la reflexión sobre responsabilidad social. Las directrices de la OCDE en materia de gestión responsable, no son un estándar adicional a los ya existentes en el marco internacional en relación con el desarrollo sostenible, sino que constituyen una integración coherente de los instrumentos preexistentes, lo que hace que se trate de una herramienta completa y adecuada en las administraciones públicas bajo la óptica del desarrollo sostenible, contemplando este concepto desde el amplio abanico de las materias ya tratadas por diferentes convenios y políticas internacionales elaboradas bajo el consenso y aprobación de diferentes grupos de interés, finalmente el Global Reporting Initiative ofrece un conjunto de indicadores en los ámbitos económico, social y ambiental que permite a las organizaciones la elaboración de reportes corporativos sobre responsabilidad social y ambiental. Este modelo ha sido utilizado para establecer un estándar mundial de empresas que han adoptado la responsabilidad social, permitiendo hacer comparaciones intra e intersectoriales (Lafuente, Viñuales, Pueyo, \& Llaría , 2003).

Un sistema de gestión de responsabilidad social se despliega a través de diferentes ámbitos o subsistemas en los que se debe integrar a las partes interesadas e involucrar internamente a todas las áreas de la organización, empleando diferentes herramientas y técnicas que facilitan su desarrollo, conocimiento, aprendizaje, retroalimentación y mejora continua (Olcese Santonja, Rodríguez, \& Alfaro, 2008). Cuadro 2.

Además de las precisiones teóricas de diferentes investigadores, en torno a la gestión de responsabilidad social en la administración pública, los Estados también se han comprometido con el desarrollo. En el año 2000, se definieron unos objetivos comunes para disminuir la pobreza extrema y la desigualdad en el mundo. La primera versión se denominó: Objetivos de Desarrollo del Milenio ODM, cuyas acciones estaban encaminadas al logro de 8 objetivos universales (ONU, Organización de las Naciones Unidas, 2001). Al concluir el período de los ODM, se evidenciaron esfuerzos mundiales, regionales, nacionales y locales. Los ODM salvaron millones de vidas y mejoraron las condiciones para muchos más, pero también es cierto que concurren logros desiguales y deficiencias en muchos aspectos. El trabajo no se ha completado y debe continuar en la nueva era del desarrollo. Es por ello que el 2015 se da continuidad a los Objetivos de Desarrollo Sostenibles ODS, que involucran en términos generales la interacción gradual del ámbito social, ambiental, económico e institucional, como un solo equilibrio solidarizado con el Desarrollo Sostenible Mundial (ONU, 2015). La interrelación directa entre los ODS y los elementos de desarrollo sostenible se presentan en el cuadro 3. 
Cuadro 2

Ámbitos y técnicas básicas de la gestión de la RSC

\begin{tabular}{|c|c|}
\hline Ámbitos de gestión & Técnicas y elementos clases de gestión de RSC \\
\hline Estratégico & $\begin{array}{l}\text { - } \quad \text { Principios y estrategias corporativas } \\
\text { - } \quad \text { Políticas y marcos generales de actuación } \\
\text { - } \quad \text { Cuadro de mando }\end{array}$ \\
\hline Estructural & $\begin{array}{l}\text { - Organización: funciones y responsabilidades } \\
\text { - Unidad responsable del sistema } \\
\text { - } \quad \text { Metodología y métodos de gestión } \\
\text { - } \quad \text { Diseño de procesos y normativas } \\
\text { - } \quad \text { Sistemas de información e instalaciones } \\
\text { - Esquema de certificación }\end{array}$ \\
\hline Operativo & $\begin{array}{l}\text { - } \quad \text { Desarrollo de proyectos } \\
\text { - } \quad \text { Registro y evidencias } \\
\text { - } \quad \text { Resultados obtenidos }\end{array}$ \\
\hline Temporalización & $\begin{array}{l}\text { - } \quad \text { Planificaciones plurianuales } \\
\text { - } \quad \text { Programas anuales } \\
\text { - } \text { Objetivos de carácter anual }\end{array}$ \\
\hline Seguimiento y evaluación & $\begin{array}{l}\text { - Informe de gestión } \\
\text { - } \quad \text { Auditorías y/o evaluaciones internas y externas } \\
\text { - } \quad \text { Revisión del sistema }\end{array}$ \\
\hline Aprendizaje y mejora & $\begin{array}{l}\text { - } \quad \text { Estudio de benchamarking } \\
\text { - } \quad \text { Proyectos de mejores prácticas } \\
\text { - } \quad \text { Planes d acción de mejora }\end{array}$ \\
\hline
\end{tabular}

Fuente: Olcese, Rodríguez \& Alfaro (2008)

\section{Cuadro 3}

Articulación de los ODS y elementos de Desarrollo Sostenible

Objetivos de Desarrollo Sostenible

Elementos de Desarrollo Sostenible

1.- Poner fin a la pobreza en todas sus formas en todo el mundo

2.- Poner fin al hambre

3.- Garantizar una vida sana y promover el bienestar para todos en todas las edades

4.- Garantizar una educación inclusiva, equitativa y de calidad y promover oportunidades de Inclusión Social aprendizaje durante toda la vida para todos

5.- Lograr la igualdad entre los géneros y empoderar a todas las mujeres y las niñas

6.- Garantizar la disponibilidad de agua y su gestión sostenible y el saneamiento para todos

7.- Garantizar el acceso a una energía asequible, segura, sostenible y moderna Protección del Medio

Ambiente

8.- Promover el crecimiento económico inclusivo y sostenible, el empleo y el trabajo decente para todos

9.- Construir infraestructuras resilientes, promover la industrialización sostenible y fomentar

10.- Reducir la desigualdad en y entre los países 


\begin{tabular}{|c|c|}
\hline Objetivos de Desarrollo Sostenible & $\begin{array}{l}\text { Elementos de } \\
\text { Desarrollo } \\
\text { Sostenible }\end{array}$ \\
\hline 11.- Lograr que las ciudades sean más inclusivas, seguras, resilientes y sostenibles & Inclusión Social \\
\hline 12.- Garantizar modalidades de consumo y producción sostenibles & $\begin{array}{l}\text { Crecimiento } \\
\text { Económico }\end{array}$ \\
\hline 13.- Adoptar medidas urgentes para combatir el cambio climático y sus efectos & \multirow[b]{3}{*}{$\begin{array}{c}\text { Protección del } \\
\text { Medio } \\
\text { Ambiente }\end{array}$} \\
\hline 14.- Conservar y utilizar sosteniblemente los océanos, los mares y los recursos marinos & \\
\hline $\begin{array}{l}\text { 15.- Gestionar sosteniblemente los bosques, luchar contra la desertificación, detener e invertir } \\
\text { la degradación de las tierras, detener la pérdida de biodiversidad }\end{array}$ & \\
\hline 16.- Promover sociedades justas, pacíficas e inclusivas & \multirow{2}{*}{ Inclusión Social } \\
\hline 17.- Revitalizar la Alianza Mundial para el Desarrollo Sostenible & \\
\hline
\end{tabular}

Fuente: Elaboración propia a partir de los ODS (ONU, 2015)

El desarrollo sostenible se ha definido como el desarrollo capaz de satisfacer las necesidades del presente sin comprometer la capacidad de las futuras generaciones; exige esfuerzos concentrados en construir un futuro inclusivo, sostenible y resiliente para las personas y el planeta. Para alcanzar el desarrollo sostenible, es fundamental armonizar elementos básicos como: el crecimiento económico, la inclusión social y la protección del medio ambiente. Estos elementos están interrelacionados y son esenciales para el bienestar de las personas y las sociedades, así como se demuestra en el cuadro 3 que antecede.

Los ODS no son jurídicamente obligatorios, sin embargo, se prevé que los países los adopten como propios y establezcan marcos nacionales para el logro de los 17 objetivos; su cumplimiento y su éxito se fundamenta en las políticas, planes y programas de desarrollo sostenible de cada país. Los países tienen la responsabilidad primordial del seguimiento y examen a nivel nacional, regional y mundial de los progresos conseguidos en el cumplimiento de los objetivos y las metas para el año 2030 (ONU, Organización de las Naciones Unidas, 2015).

A partir de la conceptualización teórica de desarrollo sostenible y responsabilidad social, y en observancia a la política social establecida en el Ecuador, desde el Plan Nacional de Desarrollo (PND) 2017-2021, el cual enmarca la búsqueda de la transparencia de la gestión pública y privada, el Estado en todo su contexto, ha impulsado un modelo de gestión que permita el desarrollo sostenible del país a largo plazo, en una relación armónica entre el ser humano y la naturaleza; equidad y justicia social, con igualdad de oportunidades, una economía social y solidaria, ecologista, como aporte significativo para el funcionamiento general y mejorar los procesos en la gestión pública, generando confianza y valor agregado en la implementación de acciones a beneficio de la ciudadanía (SENPLADES, 2017). 
Figura 2

Modelo de gestión responsable alineado

a los Objetivos de Desarrollo Sostenible

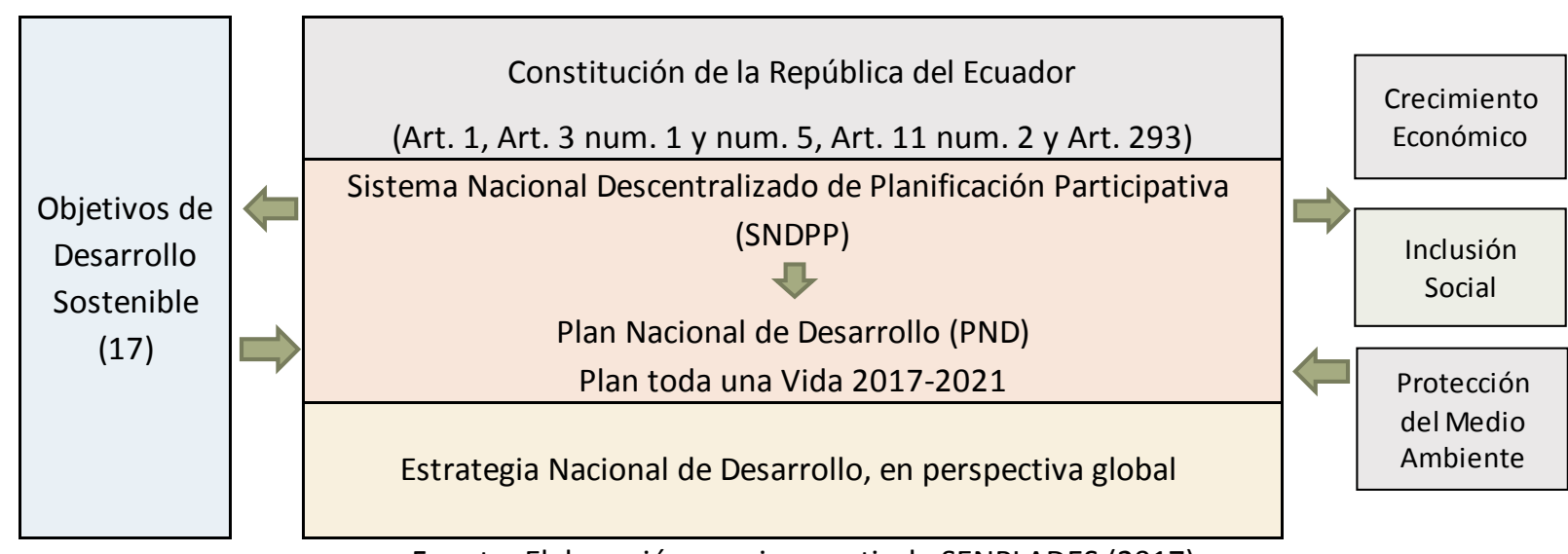

Fuente: Elaboración propia a partir de SENPLADES (2017)

De acuerdo con la figura 2, el Ecuador en su planificación de políticas públicas, tiene como prioridad la prestación de servicios a la comunidad, cumpliendo con los principios de eficacia, eficiencia y transparencia conforme lo establece la Constitución de la República del Ecuador (2008), la acción de estas actividades, los planes y programas en ejecución, metas e informes de gestión e indicadores de desempeño, corresponden a la Estrategia Nacional de Desarrollo en perspectiva global; toda esta gestión se transparenta con la difusión de la información pública a través del portal de información o página web mediante el mecanismo de rendición de cuentas a la ciudadanía, de acuerdo a lo que establece el Art. 7 de la Ley Orgánica de Transparencia y Acceso a la Información Pública LOTAIP (Congreso Nacional, 2004).

Todas las instituciones públicas se encuentran reguladas a través de un marco legal y normativo, el mismo que contiene procesos, modelos de gestión, indicadores, seguimiento y evaluación de resultados; apoyándose en tres ejes programáticos y nueve Objetivos Nacionales de Desarrollo, sobre la base de la sustentabilidad ambiental y el desarrollo territorial.

En concordancia con lo expuesto, el PND se enfoca en tres ejes, el primero, "Derechos para todos durante toda la vida", establece la protección de las personas más vulnerables, afirma la plurinacionalidad e interculturalidad, plantea el combate a la pobreza en todas sus dimensiones y todo tipo de discriminación y violencia, y garantiza los derechos de la naturaleza; el segundo eje, "Economía al servicio de la sociedad", plantea consolidar el sistema económico social y solidario, ampliar la productividad y competitividad, generar empleo digno, defender la dolarización y redistribuir equitativamente la riqueza; además busca garantizar la soberanía alimentaria y el desarrollo rural integral, y como tercer eje, "Más sociedad, mejor Estado", promueve la participación ciudadana y la construcción de una nueva ética social basada en la transparencia y la solidaridad, un Estado cercano con servicios de calidad y calidez, abierto al diálogo social permanente, así como la soberanía y la paz, posicionando estratégicamente al Ecuador en el mundo (SENPLADES, 2017); vinculados de forma directa con los ODS, permitiendo establecer estrategias conjuntas a nivel regional y global para atender las problemáticas comunes de los diferentes países; proponiendo de esta manera el desarrollo de manera integral.

El estado ecuatoriano, además de la implementación coordinada y planificada de buenas prácticas en materia de responsabilidad social, ha gestionado una serie de herramientas de gestión que acoadyuven en la adecuada ejecución de las políticas adoptadas, por mencionar un ejemplo, en el año 2010 entra en vigencia el proyecto Gobierno por Resultados (GPR), herramienta que presenta algunos objetivos como el de mejorar la ejecución del presupuesto gubernamental, mejorar la calidad de toma de decisiones, orientar al gobierno en las áreas de 
mayor urgencia de mejora, estimular la colaboración entre las instituciones del Estado, ayudar en el desmantelamiento de los procesos burocráticos, desarrollar competencias institucionales en la planificación estratégica, operativa y de gestión de resultados; entre otras, es decir, un proyecto que enmarca los enfoques de responsabilidad social, alineados a los objetivos del Plan Nacional de Desarrollo y a los ODS, involucrando los actores internos como externos (Asamblea Nacional, 2011).

\section{Metodología}

Inicialmente se trata de una investigación de tipo documental, con enfoque cualitativo ya que se apoya en la recolección de datos que sustentan su argumento en la validez y confiabilidad de las fuentes bibliográficas. Se estudiaron las diferentes definiciones y normas internacionales establecidas de algunos conceptos clave comogestión, responsabilidad social y sostenibilidad- necesarios para la comprensión posterior del análisis (Hernández, Fernández, \& Baptista, 2014). A partir de la conceptualización y correlación entre los términos objeto de estudio, se elabora un análisis comparado entre la gestión de la responsabilidad social y los objetivos de desarrollo sostenible en el sector público, por lo que la investigación se torna de tipo explicativa al esclarecer conceptos de gestión y responsabilidad social, estudiados desde la perspectiva de la administración pública. Es aquí donde se analizan las causas y efectos de la relación entre las distintas variables objeto de estudio (Bernal, 2010).

El argumento se fundamentó en la experiencia previa, el dominio del tema, la recolección de datos actualizada, diversa y confiable de fuentes bibliográficas. No existió manipulación de variables. Se trató de una observación externa sobre el acontecer corriente, por lo que se define como una investigación basada en teoría fundamentada; lo que quiere decir que la teoría proviene de los datos en sí, sin ser forzada en categorías (Hernández, Fernández, \& Baptista, 2014).

Para el alcance del objetivo principal, se realizó un análisis inicial de la gestión de responsabilidad social desde su concepción, que permitiera comprender su importancia en la gestión de la administración pública, para luego enfatizar sobre la relación directa con los objetivos de desarrollo sostenible en busca de la sostenibilidad de la administración pública.

Para finalizar, se propuso el argumento sobre la necesidad imperiosa de cambio emanada entre la gestión de la responsabilidad social que recae directamente en el administrador público. El argumento final se fundamentó en el análisis comparado entre las variables objeto de estudio, que permitieron culminar con la propuesta de estrategias propias de responsabilidad social.

Los datos utilizados para el estudio se basaron tanto en bibliografía tradicional como actualizada combinando gestión, responsabilidad social y objetivos de desarrollo sostenible en el contexto de la administración pública.

\section{Resultados}

Las definiciones teóricas permiten conocer la integración de conceptos básicos como; gestión responsable, sostenibilidad y administración pública; los mismos que alineados de manera estratégica en el modelo de gestión establecido en el Ecuador, fortalecen las prácticas de buen gobierno desde la administración de sus políticas públicas.

La propuesta del esquema está conformado por las etapas clave de gestión de la responsabilidad social, alineados de forma directa con los ámbitos y técnicas de gestión para fortalecer las buenas prácticas de responsabilidad social en la administración pública; integrando además los objetivos de desarrollo sostenible y sus elementos en los sistemas de gestión pública en el Ecuador; cada uno de ellos contempla un esquema específico en base al modelo de gestión pública como se muestra en la Figura 2. 
La entrada inicial es la estructura funcional de un proceso de gestión de responsabilidad social, integrado por la planificación, ejecución y evaluación, implantación y concienciación de una entidad pública acorde a los ámbitos de gestión estratégico, estructural, operativo, de temporalización, seguimiento y evaluación, y aprendizajes y mejora de donde se deriva el Sistema Nacional Descentralizado de Planificación Participativa (SNDPP) a través de su Plan Nacional de Desarrollo "Toda Una Vida", donde se integran dos elementos estructurales de desarrollo local: la adopción de políticas públicas incluyentes y la transversalidad de los ODS a los procesos de planificación pública.

Como salida del modelo de gestión, se encuentran los stakeholders que desde la estructura funcional de una entidad pública tienen una connotación especial por los intereses que representan, y la relevancia en el bienestar colectivo de la población. De ahí que la expectativa de la gestión pública es la de un Modelo de Gestión Responsable y Desarrollo Sostenible para mantener el equilibrio en la Administración Pública.

Figura 3

Esquema propuesto de integración de un Modelo de Gestión Responsable y Desarrollo Sostenible para el equilibrio en la Administración Pública
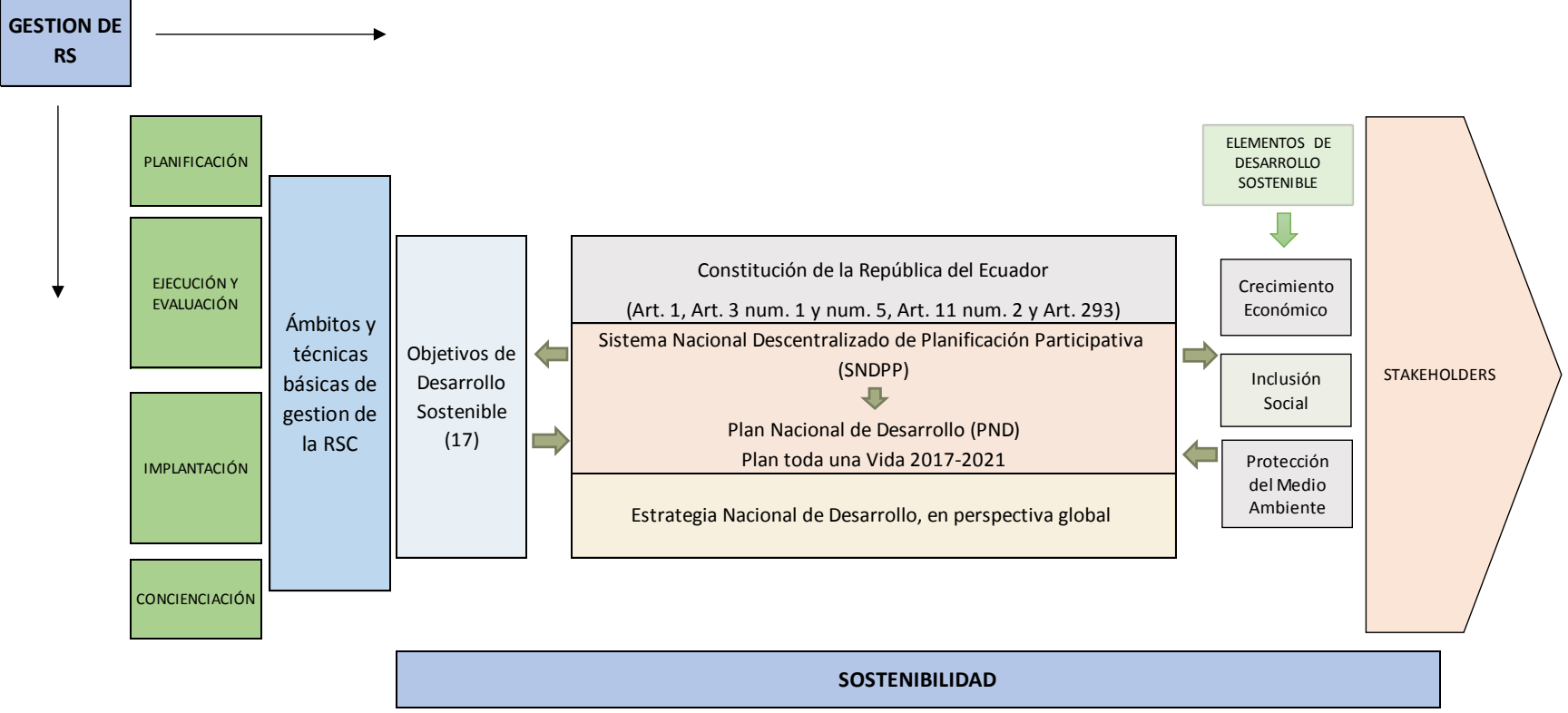

Fuente: Elaboración propia a partir de SENPLADES (2017)

\section{Conclusiones}

Una de las primeras conclusiones es la importancia que tiene para la gestión pública, en la actualidad, el identificar herramientas y estrategias de gestión que permitan enfrentar de manera eficiente los nuevos desafíos de transversalidad e intersectorialidad, asociados con la necesidad de equidad, respeto por la diversidad y desarrollo sustentable.

Se sostiene que los enfoques propuestos en el modelo de gestión, deben articularse entre sí, partiendo de una planificación que relaciona cada una de las etapas; en la primera etapa de gestión, hay que agregar que las acciones deben orientarse a la formulación de nuevos planes, programas y proyectos de desarrollo en los distintos niveles de gobierno, estableciendo modelos de desarrollo alternativos, estrategias conjuntas a nivel regional y global para atender las problemáticas comunes de los diferentes sectores.

En el contexto de sostenibilidad se destaca la importancia de articular y generar alianzas, considerando los diferentes espacios y mecanismos existentes, garantizando la inclusión de los stakeholder en forma coordinada y comprometida que permitan direccionar las acciones hacia el desarrollo de manera sostenible. 
Además, es importante identificar ciertas condiciones relacionadas con la aplicación del modelo de responsabilidad social a la gestión pública, siendo fundamental la necesaria existencia de valores y principios individuales y organizacionales que sustenten este modelo tales como la transparencia, la conciencia de los impactos, el desarrollo sustentable, etc.

\section{Referencias bibliográficas}

Albi, E., González, J., \& López, G. (1997). Gestión Pública: Fundamentos, técnicas y casos. España: Ariel Economía.

Asamblea Nacional. (28 de Diciembre de 2011). Norma de Implementación y Operación de Gobierno por Resultados. Norma de Implementación y Operación de Gobierno por Resultados, 26. Quito, Pichincha, Ecuador: Lexis Finder.

Asamblea Nacional del Ecuador. (20 de Octubre de 2008). Constitución de la República del Ecuador. Constitución de la República del Ecuador, 218. Quito, Pichincha, Ecuador: Asamblea Nacional del Ecuador.

Bernal, C. (2010). Metodología de la Investigación (Tercera ed.). Bogotá, Colombia: Pearson Educación.

Biazzo, S., \& Benardi, G. (2003). Process management practices and quality systems standards. Risk and opportunities of the new ISO 9001 certification. Business Process Management Journal, 9(2), 149-169.

Congreso Nacional. (18 de Mayo de 2004). Ley Orgánica de Transparencia y Acceso a La Información. Ley Orgánica de Transparencia y Acceso a La Información, 13. Quito, Pichincha, Ecuador: Congreso Nacional.

Cueto C., C. (2009). Investigación, desarrollo, innovación y aplicación emprendedora (I+D+i+e) de los modelos de transparencia y responsabilidad corporativa en los gobiernos y administraciones locales. Revista Análisis Local, 7-28.

Cueto, C., \& De la Cuesta, M. (2016). Enfoque e instrumentos alternativos para una gestión pública de la Responsabilidad Social Corporativa. Revista de Gestión Pública y Privada , 43-90.

Cueto, C., \& De La Cuesta, M. (2019). La Administración Pública de la Reponsabilidad Social Corporativa. Alicante: Area de Innovación y Desarrollo, S.L. doi:http://dx.doi.org/10.17993/EcoOrgyCso.2019.53

Fox, T., Ward, H., \& Howard, B. (2002). Public sector roles in strengthening corporate social responsability; a baseline study. The World Bank.

Gaete, R. (2008). Aplicaciones de la Responsabilidad Social en la nueva gestión pública. (U. N. Litoral, Ed.) Documentos y aportes en administración pública y gestión estatal(11), 1-27.

Garvin, D. (1998). The processes of organization and management. Sloan Management Review,, 33-50.

Hernández, R., Fernández, C., \& Baptista, P. (2014). Metodología de la Investigación (Sexta ed.). México: McGraw Hill.

Lafuente, A., Viñuales, V., Pueyo, R., \& Llaría , J. (2003). Responsabilidad Social Corporativa y Políticas Públicas. Laboratorio de alternativas, 1-78.

Lozano, J., Albareda, L., Tamyco, Y., Roscher, H., \& Marcuccio, M. (2005). Los gobiernos y la responsabilidad social de las empresas. Políticas públicas más allá de la regulación y la voluntariedad. Barcelona: Granica S.A. 
Maon, F., Lindgreen, A., \& Swaen, V. (2009). Designing and implementing corporate social responsability: an integrative framework grounded intheory and practice. Journal of Business Ethics, 71-89.

Navarro, A., Ruiz, M., De Los Rios, A., \& Tirado, P. (2014). La resposabilidad social corporativa en los gobiernos locales; el caso de Reino Unido e Irlanda. Innovar, 4(54), 89-105. doi:http://dx.doi.org/10.15446/innovar.v24n54.46446

Olcese Santonja, A., Rodríguez, M., \& Alfaro, J. (2008). Manual de la empresa responsable y sostenible. Conceptos, ejemplos de herramientas de la responsabilidad social corporativa o de la empresa. Madrid: McGraw-Hill Interamericana.

ONU, O. d. (2001). Organización de las Naciones Unidas. Obtenido de Organización de las Naciones Unidas: https://www.un.org/es/chronicle/article/la-importancia-de-los-objetivos-de-desarrollo-del-milenio-elliderazgo-de-las-naciones-unidas-en-el

ONU, O. d. (2015). Organización de las Naciones Unidas. Obtenido de Organización de las Naciones Unidas: https://www.un.org/sustainabledevelopment/es/sustainable-development-goals/

SENPLADES, S. N. (22 de Septiembre de 2017). Plan Nacional de Desarrollo 2017-2021. Plan Nacional de Desarrollo 2017-2021, 148. Quito, Pichincha, Ecuador: SENPLADES.

Suarez-Barraza, M., \& Ramis-Pujol, J. (2008). Aplicación y Evolución de la Mejora Continua de Procesos en la Administración Pública. Journal of Globalization, Competitiveness \& Governability, 74-86.

Tomassini , L., \& Armijo, M. (2002). Reforma y modernización del Estado. Experiencias y desafío. Santiago de Chile: LOM.

Tomassini, L. (1994). La Reforma del Estado y las Políticas Públicas. Santiago de Chile: Universidad de Chile. Centro de Análsis de Políticas Públicas.

Zenck, M., Rios, I., Pogo, L., \& Cueto, C. (2017). Análisis de la transparencia sobre las políticas locales de responsabilidad social en Ecuador: Estudio de los casos de Quito, Guayaquil y Machala. Revista Iberoamericana de Contabilidad de Gestión, XV(29), 23.

Esta obra está bajo una Licencia Creative Commons Attribución-NoCommercial 4.0 International

\section{(cc) EY-NO}

